\title{
Frizzled 3 and Frizzled6 Cooperate with Vangl2 to Direct Cochlear Innervation by Type II Spiral Ganglion Neurons
}

\author{
Satish R. Ghimire ${ }^{1}$ and Michael R. Deans ${ }^{1,2}$ \\ ${ }^{1}$ Department of Neurobiology \& Anatomy, University of Utah School of Medicine, Salt Lake City, Utah 84132, and 2Department of Surgery, Division of \\ Otolaryngology, University of Utah School of Medicine, Salt Lake City, Utah 84112
}

Type II spiral ganglion neurons provide afferent innervation to outer hair cells of the cochlea and are proposed to have nociceptive functions important for auditory function and homeostasis. These neurons are anatomically distinct from other classes of spiral ganglion neurons because they extend a peripheral axon beyond the inner hair cells that subsequently makes a distinct 90 degree turn toward the cochlear base. As a result, patterns of outer hair cell innervation are coordinated with the tonotopic organization of the cochlea. Previously, it was shown that peripheral axon turning is directed by a nonautonomous function of the core planar cell polarity (PCP) protein VANGL2. We demonstrate using mice of either sex that $F z d 3$ and $F z d 6$ similarly regulate axon turning, are functionally redundant with each other, and that $F z d 3$ genetically interacts with Vangl 2 to guide this process. FZD3 and FZD6 proteins are asymmetrically distributed along the basolateral wall of cochlear-supporting cells, and are required to promote or maintain the asymmetric distribution of VANGL2 and CELSR1. These data indicate that intact PCP complexes formed between cochlear-supporting cells are required for the nonautonomous regulation of axon pathfinding. Consistent with this, in the absence of PCP signaling, peripheral axons turn randomly and often project toward the cochlear apex. Additional analyses of Porcn mutants in which WNT secretion is reduced suggest that noncanonical WNT signaling establishes or maintains PCP signaling in this context. A deeper understanding of these mechanisms is necessary for repairing auditory circuits following acoustic trauma or promoting cochlear reinnervation during regenerationbased deafness therapies.

Key words: auditory; axon; cochlea; frizzled; planar cell polarity; spiral ganglia

Significance Statement

Planar cell polarity (PCP) signaling has emerged as a complementary mechanism to classical axon guidance in regulating axon track formation, axon outgrowth, and neuronal polarization. The core PCP proteins are also required for auditory circuit assembly, and coordinate hair cell innervation with the tonotopic organization of the cochlea. This is a non-cell-autonomous mechanism that requires the formation of PCP protein complexes between cochlear-supporting cells located along the trajectory of growth cone navigation. These findings are significant because they demonstrate how the fidelity of auditory circuit formation is ensured during development, and provide a mechanism by which PCP proteins may regulate axon outgrowth and guidance in the CNS.

\section{Introduction}

Intercellular signals relayed by planar cell polarity $(\mathrm{PCP})$ proteins contribute to an array of developmental events, including axon

Received July 20, 2019; revised Aug. 20, 2019; accepted Aug. 23, 2019.

Author contributions: S.R.G. and M.R.D. designed research; S.R.G. and M.R.D. performed research; S.R.G. wrote the first draft of the paper; M.R.D. edited the paper; M.R.D. wrote the paper.

This work was supported by National Institute on Deafness and Other Communication Disorders Grant R21DC015635 and University of Utah Research Incentive Seed Grant. We thank Shinichi Aizawa, Andrew Groves,

Charles Murtaugh, Rejji Kuruvilla, and Jeremy Nathans for providing mouse lines used in this study; and Orvelin Roman Jr and Sarah Siddoway for assistance in animal colony management and genotyping.

The authors declare no competing financial interests.

Correspondence should be addressed to Michael R. Deans at michael.deans@utah.edu.

https://doi.org/10.1523/JNEUROSCI.1740-19.2019

Copyright $\odot 2019$ the authors outgrowth and guidance, and the establishment of planar polarity (Zou, 2004; Wang and Nathans, 2007; Goodrich and Strutt, 2011; Tissir and Goffinet, 2013). In the murine cochlea, planar polarity is guided by an intercellular signaling complex composed of the PCP proteins Frizzled (FZD3/6) (Wang et al., 2006), Van Gogh-like (VANGL1/2) (Montcouquiol et al., 2003; Torban et al., 2008), and CELSR1 (Curtin et al., 2003). Complex formation requires FZD3/6 and VANGL1/2 localization at opposite sides of individual cells so that their extracellular domains appose each other at junctions between neighbors, and this complex is maintained by homophilic CELSR1 binding across intercellular space. In Drosophila, the analogous complex relays polarity information through Fzd to Van Gogh signaling (Wu and Mlodzik, 
2008) or homophilic signaling by the CELSR1 ortholog Flamingo/Starry Night (Struhl et al., 2012). Vertebrate PCP proteins similarly relay polarity information within epithelia (Mitchell et al., 2009; Sienknecht et al., 2011; Stoller et al., 2018). While mechanisms initiating PCP may vary, an emerging consensus for vertebrates is that noncanonical WNT signaling promotes initial protein asymmetries that are relayed between neighbors by the PCP complex (Yang, 2012; Yang and Mlodzik, 2015).

PCP signaling is not restricted to epithelia, and PCP proteins contribute to axon outgrowth (Fenstermaker et al., 2010; Morello et al., 2015), pathfinding (Lyuksyutova et al., 2003; Shafer et al., 2011; Hua et al., 2013; Onishi et al., 2013), and neuronal migration (Qu et al., 2010; Glasco et al., 2012; Sittaramane et al., 2013; Davey et al., 2016). Phenotypic analyses suggest that different molecular mechanisms are used for these processes in different developmental contexts. For example, commissural neurons extend an axon across the ventral midline that turns anteriorly in response to a WNT gradient (Zou, 2004; Onishi and Zou, 2017). In the absence of Vangl2 (Shafer et al., 2011), Frizzled3 (Lyuksyutova et al., 2003), or Celsr3 (Onishi et al., 2013), commissural axons cross the midline but turn incorrectly. FZD and VANGL2 regulation of filopodial dynamics in the growth cone (Onishi et al., 2013) indicates that this is a cell-autonomous mechanism. In contrast, VANGL2 acts non-cell-autonomously to guide the peripheral axon of Type II spiral ganglion neurons (SGNs) in the cochlea (Ghimire et al., 2018). FZD3 and CELSR also act noncell-autonomously to promote the formation of long axon tracks (Morello et al., 2015; Feng et al., 2016); however, this does not require VANGL1/2 (Qu et al., 2014) and suggests that an intact intercellular PCP complex is not required for all axon guidance events. These analyses demonstrate that PCP proteins contribute to multiple guidance events but suggest they act through mechanisms that differ from PCP signaling in epithelia.

In the developing cochlea, VANGL2 ensures that the peripheral axons of Type II SGNs complete a $90^{\circ}$ turn toward the cochlear base before innervating the outer hair cells (OHCs) (Ghimire et al., 2018). SGNs are bipolar relay neurons that convey auditory information and consist of two major subtypes. Type I SGNs innervate inner hair cells (IHCs), comprise 90\%$95 \%$ of the SGN population, and transmit the salient features of sound. Type II SGNs comprise the remainder and are thought to be neuroprotective rather than sensory because they resemble nociceptive c-fibers (Flores et al., 2015; Liu et al., 2015; Maison et al., 2016). Peripheral axon turning is dependent upon VANGL2 and CELSR1, and many fibers turn incorrectly toward the cochlear apex in these mutants. Here we demonstrate that Fzd3 and Vangl2 act in a common pathway to guide axon turning and that $F z d 3$ and Fzd6 are functionally redundant. Reducing WNT signaling also disrupts turning, likely as a result of mislocalized core PCP protein distribution. Based upon these results, we propose that a PCP signaling axis established in the cochlear epithelia directs the peripheral axons of Type II SGNs to turn toward the cochlear base.

\section{Materials and Methods}

Mouse strains and husbandry. All mice were housed at the University of Utah under Institutional Animal Care and Use Committee approved guidelines, and individual lines were maintained by backcross to B6129SF1/J hybrid females and genotyped using allele-specific PCRs (available upon request). For timed breeding and tissue staging, noon on the day in which vaginal plug was seen was considered embryonic day 0.5 (E0.5), and the day litters were born was considered postnatal day 0 (P0). Mice from both sexes were used for experimentation, with the exception of analyses of Porcn mutants because the Porcn gene is located on the $\mathrm{X}$-chromosome. $\mathrm{Fzd3}^{-/-} ; \mathrm{Fzd6}^{-/-}$double mutants and littermate controls were generated by intercrossing $\mathrm{Fzd3}^{+/-} ; \mathrm{Fzd6}^{+/-}$males and females derived from the Fzd3 and Fzd6 KO lines. Emx2-Cre;Fzd3 CKOs and Cre-positive littermate controls were generated on a Fzd6 KO background by crossing Emx2-Cre;Fzd3 ${ }^{+/-} ; \mathrm{Fzd6}^{+/-}$males with Fzd3 ${ }^{\text {floxed }}$; $\mathrm{Fzd6}^{-1-}$ females. Pax2-Cre ${ }^{+}$; Vangl2 CKOs and Cre-positive littermate controls were generated on a Fzd3 KO background by crossing Pax2-Cre; Vangl2 $^{+/-} ; \mathrm{Fzd3}^{+/-}$male mice with Vangl2 ${ }^{\text {floxed }} ; \mathrm{Fzd3}^{+/-}$females. Pax2Cre;ROR2;Vangl2 CKOs and Cre-positive littermate controls were generated by crossing Pax2-Cre ${ }^{+} ; V_{\text {Vangl }}{ }^{+/-} ; R O R 2^{\text {LoxP/+ }}$ male mice with Vangl2 ${ }^{\text {floxed }} ;$ ROR2 $2^{\text {floxed }}$ female mice. Pax $2-\mathrm{Cre}^{+} ; \mathrm{Fzd}_{3} 3^{3 \mathrm{XHA} /+}$ tissue was generated for the study of transgenic FZD3 protein localization by crossing Pax2-Cre ${ }^{+}$male mice with Fzd $3^{3 X H A /+}$ female mice. The Wnt5a $\mathrm{KO}$ allele was generated by crossing $W n t 5 a^{\text {floxed }}$ mice (Ryu et al., 2013) with $C M V$-Cre (Schwenk et al., 1995) to delete coding sequence exon 2 and then backcrossed with B6129SF1/J hybrid mice to obtain Crenegative $W n t 5 a$ heterozygotes. Wnt5a $a^{+/-}$males and females were intercrossed to generated Wnt5a KOs. Pax2-Cre;Porcn CKOs were generated by crossing Pax2-Cre ${ }^{+}$male mice with Porcn ${ }^{\text {LoxP/WT }}$ female mice. Since the Porcn gene is located on the $\mathrm{X}$-chromosome, the analysis was restricted to males with $\mathrm{Pax}_{2}-\mathrm{Cre}^{+} ; \mathrm{Porcn}^{\text {Loxp/Y }}$ embryos used as experimental and Pax2-Cre ${ }^{+;}$Porcn ${ }^{\text {WT/Y }}$ embryos as littermate controls. Male pups were identified by genotyping for the male-specific Sry allele. Emx2Cre mice (Kimura et al., 2005) were provided by S. Aizawa (Riken Institute). Fzd3 KO (Wang et al., 2002), Fzd 6 KO (Guo et al., 2004), and Fzd3 CKO (Hua et al., 2013) mice were provided by J. Nathans (John Hopkins University). Pax2-Cre mice (Ohyama and Groves, 2004) were provided by A. Groves (Baylor College of Medicine). Porcn CKO mice (Barrott et al., 2011) were provided by C. Murtaugh (University of Utah). Wnt5a CKO line (Ryu et al., 2013) was provided by R. Kuruvilla (John Hopkins University). Generation of Vangl2 ${ }^{\text {floxed }}$ and Vangl2 KO lines has been described previously (Yin et al., 2012; Copley et al., 2013). B6129SF1/J hybrid (strain \#101043), CMV-Cre (strain \#006054) (Schwenk et al., 1995), Fzd3 $3^{3 X H A /+}$ (strain \#023841 (Hua et al., 2014b), and ROR2 mice (strain \#018354) (Ho et al., 2012) were purchased from The Jackson Laboratory.

Immunofluorescence. Inner ear tissues were fixed using 4\% PFA in 67 mM Sorensons' phosphate buffer, $\mathrm{pH}$ 7.4, for $2 \mathrm{~h}$ on ice. For wholemount immunofluorescent labeling, cochlea were microdissected and detergent permeabilized using a blocking solution (5\% normal donkey serum, $1 \%$ BSA, PBS) supplemented with $0.5 \%$ Triton X-100 with the exception of HA immunostaining where 3\% BSA prepared in PBS was used as the blocking solution. Primary antibodies and Phalloidin AlexaFluor-488 (Invitrogen A12379) were diluted in blocking solution supplemented with $0.1 \%$ Tween 20 and incubated overnight at $4{ }^{\circ} \mathrm{C}$. Tissue was washed thoroughly using PBS with $0.05 \%$ Tween 20 and incubated with species-specific Alexa-conjugated or Cy3-conjugated secondary antibodies (Jackson ImmunoResearch Laboratories, 705-165147, 705-605-147, 706-165-148, 711-165-152, 711-545-152, 711-605$152,712-605-150)$, before mounting for fluorescence microscopy using Prolong Gold (Thermo Fisher Scientific, P36930). Fluorescent images were acquired by structured illumination microscopy using the Carl Zeiss Axio Imager M.2 with ApoTome.2 attachment and Axiocam 506 mono camera. Images were processed using Carl Zeiss Zen software, and figures were prepared using with Adobe Illustrator. Commercial antibodies used in this study are as follows: rabbit anti- $\beta$-galactosidase (Millipore, AB986, 1:500), goat anti-FZD6 (R\&D Systems, AF1526, dil 1:200), rabbit anti-HA (Cell Signaling Technology, C29F4, 1:1000), rat anti-HA (Sigma-Aldrich, 11867423001, dil 1:200), and rabbit antiNeurofilament H (NF200) (Millipore, AB1989, dil1:1500). Guinea pig anti-CELSR1 (Devenport and Fuchs, 2008) (dil1:1000) was a gift from D. Devenport (Princeton University), and rabbit anti-VANGL2 (Montcouquiol et al., 2003) (dil1:1500) was a gift from M. Montcouquiol (Universite' Bordeaux).

ISH. Whole heads from E16.5 embryos were fixed overnight using 4\% PFA prepared in PBS, $\mathrm{pH} 7.4$, at $4^{\circ} \mathrm{C}$, cryoprotected in $30 \%$ sucrose before freezing in Neg50 (Richard Allan Scientific) blocks, and sectioned at $16 \mu \mathrm{m}$ using a CM3050 cryostat (Leica Microsystems). For conven- 


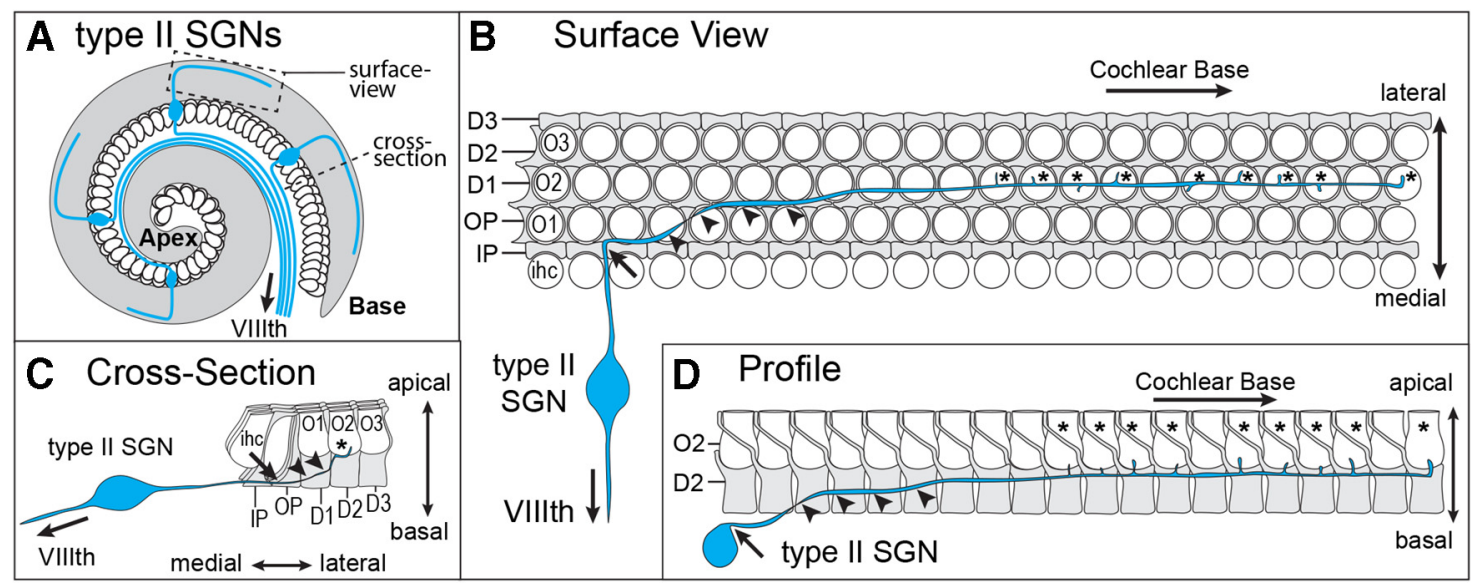

Figure 1. Peripheral axons from Type II SGNs project toward the cochlear base. A, Schematic diagram of the mouse cochlea illustrating the anatomy of the Type II SGN with a peripheral axon that innervates the cochlea and turns toward the cochlear base, and a central axon that exits the inner ear through the VIllth cranial nerve. $\boldsymbol{B}$, Surface view of the mouse organ of Corti, illustrating the morphology of the Type II SGN peripheral axon relative to the apical surfaces of hair cells and supporting cells. The peripheral axon extends beyond the single row of IHCs (ihc) and completes a $90^{\circ}$ turn (arrow) toward the cochlear base before ascending apically (arrowheads) and extending branches to form afferent synapses (asterisk) with OHCs. Turning frequently occurs after the axon has passed between the basolateral junctions formed between neighboring supporting cells, as illustrated here for IPCs. C, Cross-section of the organ of Corti, illustrating the position of axon turning (arrow) amid the basolateral boundaries of pillar cells before apical ascension (arrowheads) and synapse formation (asterisk). D, Profile of a single row of OHCs (02) and Deiters' cells (D2), illustrating the site of ascension (arrowheads) of the peripheral axon after turning (arrow) and before synapse formation (asterisk). IHCs, first (01), second (02), and third (03) rows of 0HCs, IPCs, 0PCs, first (D1), second (D2), and third (D3) row of Deiters' cells.

tional ISH hybridization (detailed protocols available upon request), sections were hybridized with digoxigenin-labeled antisense probes and detected using alkaline phosphatase-conjugated anti-digoxigenin antibodies (Roche Diagnostics, catalog \#11093274910, dil 1:2000). Following histochemical detection, slides were coverslipped and imaged using an Axio Imager M.2 and Axiocam 105 color camera (Carl Zeiss). Probe template for $F z d 3$ was generated by PCR amplification of embryonic cDNA using the following primer pair, 5'TGAACCATTATGACCAA CAGACT3' and 5'CTCGCACGTGCAGAAAGGAAT3', followed by TOPO cloning into the pCRII-TOPO vector. This $F z d 3$ probe detects an exon of the Fzd3 gene flanked by LoxP sites and hence is deleted in Fzd3 CKOs.

Quantification of peripheral axon turning defects. Type II SGN peripheral axons were identified for quantification based upon morphological criteria following Neurofilament H (NF200) immunolabeling. Fiber turning was evaluated in the region between the termination of Type I fibers at the IHCs and the first outer spiral bundle beneath OHC1. For E18.5 cochlear samples, evaluation was restricted to the basal turn of the cochlea ( $25 \%$ cochlear length from basal end) as Type II peripheral axon turning occurs in a gradient from the cochlear base to apex, and fibers in apical locations had not yet turned at this stage. For all other ages, turning was accessed at three locations corresponding to $25 \%, 50 \%$, and $75 \%$ the cochlear length.

Experimental design and statistical analyses. Graphing and statistical analyses were conducted using Microsoft Excel. Student's $t$ test (unpaired with equal variance) was used to determine the statistical significance of differences between mutant and littermate control pairs as indicated. Significance was declared in circumstances where $p \leq 0.05$. A minimum of 3 animals were used for each genotype as detailed in figure legends, with a single cochlea from each used for imaging and quantification. Data are mean $\pm \mathrm{SD}$.

\section{Results}

The peripheral axons of Type II spiral ganglion neurons always project toward the cochlear base

SGNs are bipolar relay neurons located within the inner ear that project a central axon to the auditory hindbrain and a peripheral axon to the organ of Corti, a narrow sensory epithelium spiraling along the length of the cochlea, which contains the sensory receptor hair cells that convert the mechanical stimuli of sound into neuronal activity (Fig. 1A). The organ of Corti also contains dis- tinct populations of supporting cells that surround the hair cells, provide structural and trophic support, and include the inner pillar cells (IPCs) and outer pillar cells (OPCs) and Deiters' cells. Type II SGNs selectively innervate the three rows of OHCs and extend a peripheral axon past the single row of IHCs that subsequently makes a $90^{\circ}$ turn toward the cochlear base (Fig. $1 B$ ) (Zhang and Coate, 2017). During outgrowth, Type II SGN peripheral axon turning occurs after the growth cone passes between the basolateral surfaces of cochlear-supporting cells. This happens most frequently after passage between IPCs, which are the first supporting cell class encountered by the growth cone, although turning may also occur among the OPCs and Deiters' cells (Fig. $1 B$ ). In some instances, a neuron may turn several times in a stepwise fashion throughout its trajectory; however, outgrowth is always directed toward the cochlear base. Following turning, peripheral axons gradually ascend apically toward the OHCs, joining other Type II axons in one of three outer spiral bundles, and extending branches to contact individual $\mathrm{OHCs}$ within a single row (Fig. $1 C, D$ ). Thus, turning occurs between the basolateral walls of cochlear-supporting cells in an area that is spatially distinct from OHCs (Fig. 1D).

\section{Frizzled 3 and Frizzled6 are functionally redundant in growth cone turning}

The core PCP proteins VANGL2 and CELSR1 are required for peripheral axon guidance in the developing cochlea, and Type II SGN fibers frequently turn incorrectly and project toward the cochlear apex the mice lacking these genes. FZD3 is also required for axon outgrowth and growth cone navigation in other contexts (Hua et al., 2013, 2014a); however, the frequency of Type II SGN turning errors is significantly less in Fzd3 KO than in Vangl2 or Celsr1 KO mice (Ghimire et al., 2018). One explanation is genetic redundancy or compensation by other FZD receptors. Consistent with this, Fzd3 and Fzd6 have a redundant function in controlling neural tube closure and stereociliary bundle orientation (Wang et al., 2006), and Fzd3 can rescue the Fzd6 PCP phenotypes in skin (Hua et al., 2014b). FZD3 distribution can be visualized via immunofluorescent labeling of HA in the mouse using a Fzd3 CKO 
line in which the extracellular domain of FZD3 is tagged by an HA epitope (Hua et al., 2013). In the organ of Corti of the cochlea, FZD3-HA is enriched at hair cell to supporting cell junctions near their apical surfaces in a pattern similar to FZD6 (Fig. 2A,B). FZD3-HA and FZD6 are also present along the basolateral sides of cochlear-supporting cells and appear enriched at cellular junctions (Fig. 2C,D). However, at this location, the cellular boundaries containing FZD3-HA and FZD6 are aligned parallel to the mediolateral axis of the cochlea and thus perpendicular to FZD3-HA and FZD6 protein on the apical surface (Fig. 2A,B). This labeling accurately reflects FZD3 and FZD6 distribution in supporting cells because FZD3-HA and FZD6 immunofluorescence is lost in WT and Fzd6 KO tissue, respectively (Fig. 2E,F).

Since FZD proteins are asymmetrically distributed in single cells in other contexts, an outstanding question is whether FZD3 distribution is also asymmetric in supporting cells and whether it is enriched on the basolateral side facing the cochlear apex or the cochlear base. A reporter mouse in which a 3xHA-tagged FZD3 transgene is expressed from the ubiquitin $\mathrm{B}(U b b)$ locus under the control of a Cre-dependent regulatory cassette allows transgenic 3xHA-FZD3 protein distribution to be evaluated in individual cells (Fig. 2G) (Hua et al., 2014b). In these mice, Cremediated recombination excises the lac $Z$ gene, and its product $\beta$-galactosidase can be used to distinguish Cre-negative cells from Cre-positive cells expressing 3xHA-FZD3 that lack $\beta$-galactosidase. Activation of this reporter by Pax2-Cre is inefficient and does not occur in all cells. As a result, a mosaic pattern of $3 \mathrm{xHA}-\mathrm{FZD} 3$ expression can be generated that is predicted to match the asymmetric distribution of endogenous FZD3. In these mice, 3xHA-FZD3 is distributed along the side of individual cells facing the cochlear base (Fig. $2 H$ ). VANGL2 is also asymmetrically distributed along the basolateral wall of supporting cells but is enriched on the side of individual cells facing the cochlear apex (Ghimire et al., 2018). Thus, at the boundaries between cochlearsupporting cells, FZD3 is positioned opposite of VANGL2 consistent with the relative distribution reported for these proteins at intercellular junctions on the apical surface (Wang et al., 2006; Giese et al., 2012). CELSR1 is also present between cochlearsupporting cells (Ghimire et al., 2018), indicating that an intact PCP signaling complex may function at the intercellular junctions between the basolateral surfaces of these cells.

Functional redundancy between $\mathrm{Fzd} 3$ and $\mathrm{Fzd} 6$ was evaluated in SGN turning by generating Fzd3;Fzd6 double KOs (dKOs). Similar to prior reports, the loss of both genes could lead to craniorachischisis (Fig. 3B); however, this neural tube defect was only found in two Fzd3;Fzd6 dKOs (2 of 7). Other mutants had exencephaly ( 2 of 7) or normal body axes ( 3 of 7 ), but all dKOs had the looped tail characteristic of other core PCP mutants (Fig. $3 B-D)$. Since the mutant alleles were carried on mixed genetic backgrounds, this phenotypic variability is likely due to strain variation. However, regardless of the severity of the neural tube defect, all cochleae from Fzd3; Fzd6 dKOs had IHCs with misoriented stereociliary bundles (Fig. $3 A^{\prime}-D^{\prime}$ ), indicating that planar polarity phenotypes are fully penetrant within the cochlea of these mice. Consistent with this observation, Type II peripheral axon turning was severely disrupted in all cochlea from $F z d 3 ; F z d 6$ dKOs with an average of $52.7 \%$ of fibers turning incorrectly toward the cochlear apex (Fig. $3 H, I$ ). Due to the neural tube defect, embryos were collected at E18.5. At this age, fibers have started to turn but have not reached their full length or formed the three outer spiral bundles beneath the OHCs and could only be analyzed in the basal turn (a location 25\% of the cochlear length measured from the base). The single mutants generated from
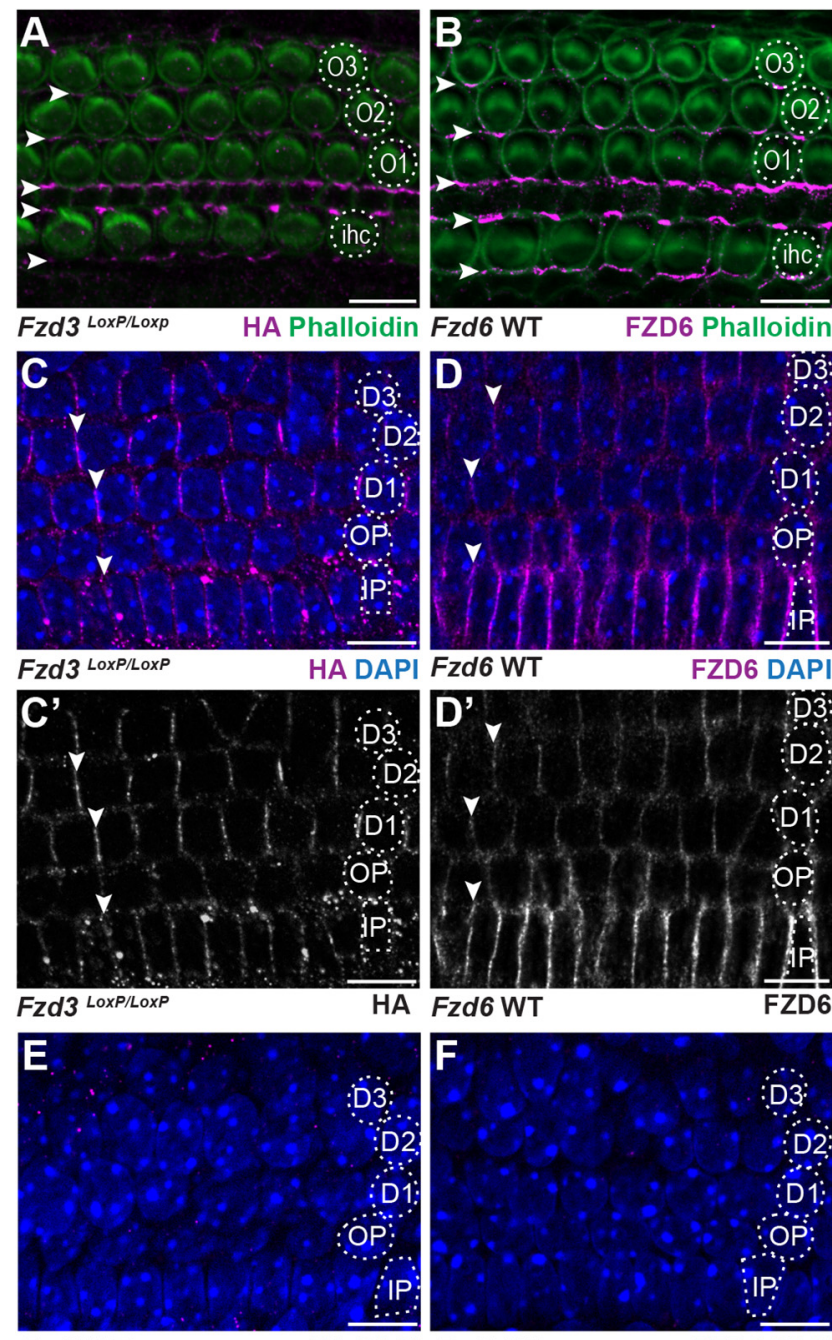

A Fzd6 WT
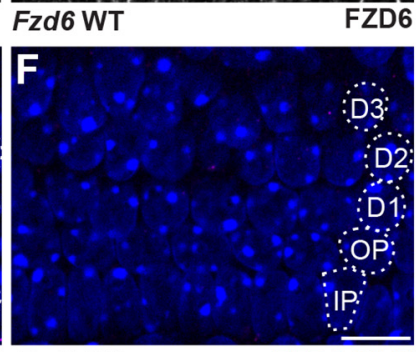

G
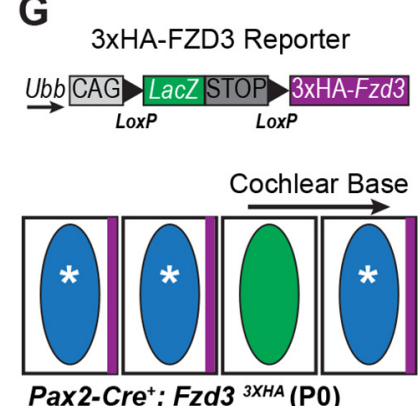

$\mathrm{H}$

FZD6 DAP

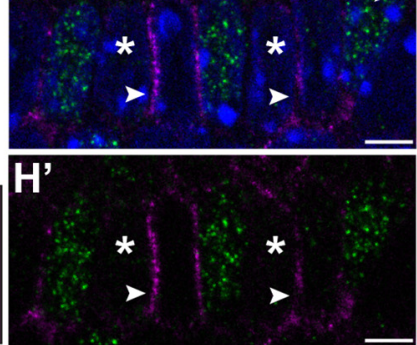

Pax2-Cre ${ }^{+}$Fzd3 ${ }^{3 \times H A}$ (PO)

HA $\beta$-Galactosidase DAPI

Figure 2. FZD3 and FZD6 are asymmetrically distributed along the basolateral sides of cochlear-supporting cells. $A, \mathrm{HA}$ immunofluorescence at $\mathrm{PO}$ is enriched at hair cell to supporting cell junctions on the apical surface of the organ of Corti in a Fzd3 CKO mouse in which endogenous FZD3 contains an HA epitope tag (arrowheads). $\boldsymbol{B}$, FZD6 protein is similarly enriched at hair cell to supporting cell junctions (arrowheads). $\boldsymbol{C}_{1} \boldsymbol{C}^{\prime}$, FZD3-HA and $\mathbf{D}, \mathbf{D}^{\prime}$, FZD6 are also enriched along the basolateral surfaces of supporting cells at supporting cell to supporting cell junctions (arrowheads). E, Specificity of the HA antibody demonstrated by immunolabeling the basolateral surfaces of supporting cells in WT tissue and $(\boldsymbol{F})$ specificity of the FZD6 antibody demonstrated in Fzd6 K0 tissue. G, Schematic illustration of a Cre-dependent 3XHA-Fzd3 transgenic reporter and predicted distribution of the 3 XHA-FZD3 protein product (magenta) in adjacent Cre-positive (blue nuclei, asterisk) and (re-negative (green nuclei) cells. $\boldsymbol{H}_{\boldsymbol{1}} \boldsymbol{H}^{\prime}, 3 \mathrm{XHA-FZD3}$ expression and localization (arrowheads) in the Pax2-Cre ${ }^{+}$IPCs (asterisk) on the side facing the cochlear base. $\beta$-galactosidase immunolabel (green) marks (re-negative cells, which do not express the FZD3 reporter. IHCs, first (01), second (02), and third (03) row $0 H C S, I P C s, 0 P C s$, first (D1), second (D2), and third (D3) row Deiters' cells. All images are of P0 tissue. Scale bars: $A-F$, $10 \mu \mathrm{m} ; \boldsymbol{H}, 5 \mu \mathrm{m}$. 

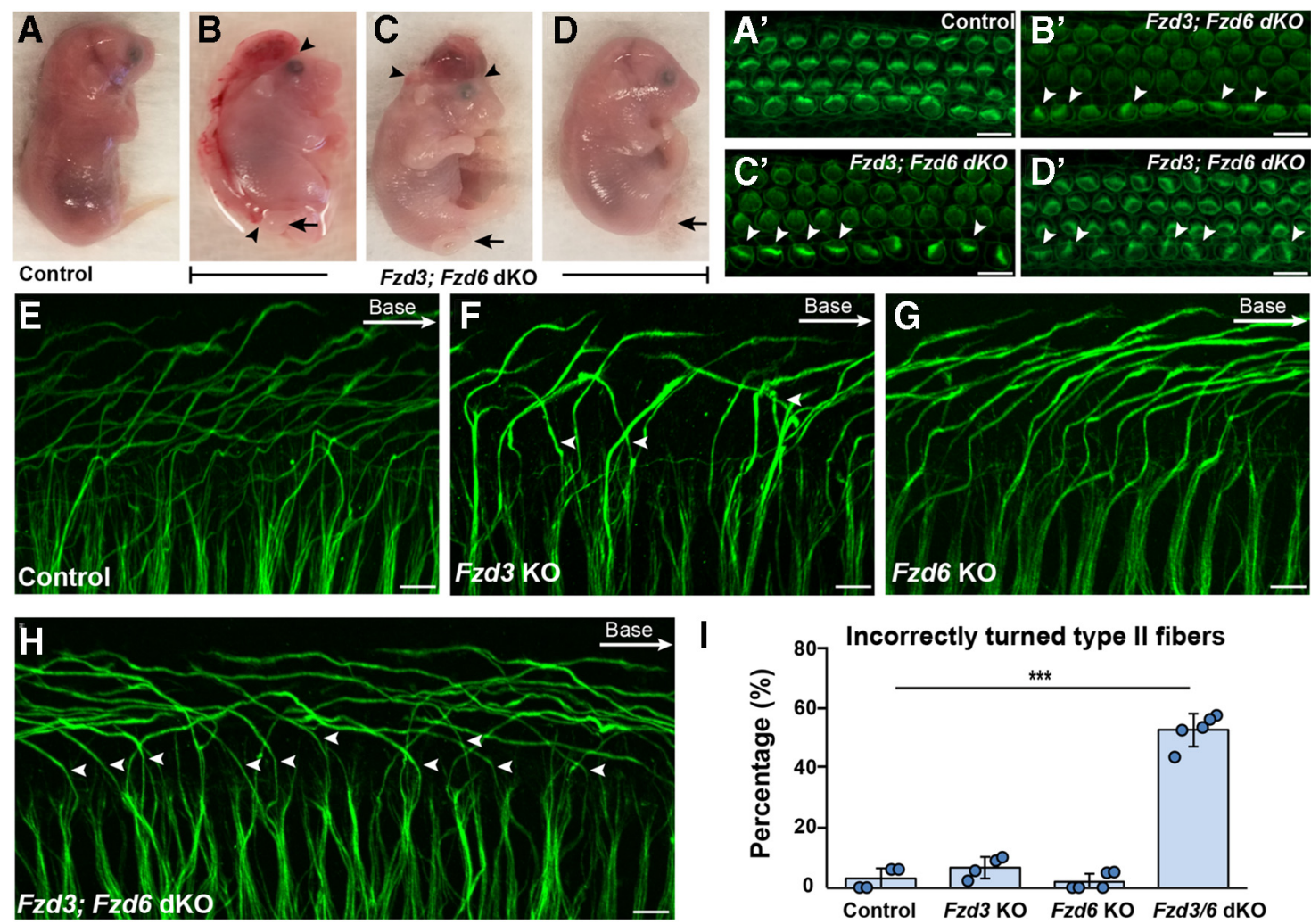

Figure 3. Fzd3 and Fzd6 act redundantly to direct Type II SGN turning. A-D, All Fzd3;Fzd6 dK0s have characteristic looped tails (arrows) but variable neural tube defects ranging from craniorachischisis $(\boldsymbol{B})$ to exencephaly $(\boldsymbol{C})$ to normal body axes $(\boldsymbol{D})$. $\boldsymbol{C}, \boldsymbol{D}$, Arrowheads indicate the anterior and posterior extent of the neural tube defect. $\boldsymbol{A}^{\prime}-\boldsymbol{D}^{\prime}$, Phalloidin staining of hair cell stereociliary bundles from cochlea dissected from $F z d 3 ; F z d 6 \mathrm{dKO}$ embryos (corresponding to $B, C, D$ ) shows that all have misoriented bundles (arrowheads) regardless of the severity of the neural tube defect. $\boldsymbol{E}-\boldsymbol{H}, \mathrm{NF} 200$ immunolabeling of Type II peripheral axons shows that the majority project toward the cochlear base in littermate controls $(\boldsymbol{E})$, Fzd3 $\mathrm{KOs}(\boldsymbol{F})$, and Fzd6 K0s $(\boldsymbol{G})$, whereas in $F z d 3 ; F z d 6 \mathrm{dKOs},(\boldsymbol{H})$ many turn incorrectly toward the cochlear apex (arrowheads). $\boldsymbol{I}$, The average frequency of incorrectly turned Type II peripheral axons in dK0, single K0, and littermate control animals. Data are mean \pm SD with overlying circles reporting to the frequency of turning errors for individual mice. All images and quantification were collected from the basal turn of E18.5 cochlea with a single cochlea analyzed from each mouse: Control ( $n=4,159$ axons), Fzd3 KO ( $n=4,178$ axons), Fzd6 KO ( $n=5,240$ axons), Fzd3; Fzd6 dKO ( $n=5,231$ axons). ${ }^{* * *} p \leq 0.001, F z d 3 ; F z d 6$ dKOs versus littermate controls (Student's $t$ test). Scale bars, $10 \mu \mathrm{m}$.

these intercrosses had normal patterns of Type II SGN innervation comparable with littermate controls (Fig. 3E-G,I). Thus, similar to prior reports for auditory hair cells (Wang et al., 2006), $F z d 3$ and Fzd6 are functionally redundant during the course of cochlear innervation.

Frizzled 3 and Frizzled6 functional redundancy occurs in the organ of Corti

The core PCP protein VANGL2 directs Type II peripheral axon turning through a non-cell-autonomous mechanism that does not require VANGL2 function in the migrating growth cone (Ghimire et al., 2018). One possibility is that the polarized distribution of VANGL2 and FZD3/6 in organ of Corti-supporting cells provides directional cues to the incoming growth cone or promotes the planar polarized distribution of classical axon guidance cues. To determine whether Fzd3 and Fzd6 contribute to organ of Corti planar polarity during innervation, Fzd3 CKOs were generated on the Fzd6 KO background using Emx2-Cre to remove Fzd3 from the cochlear duct but not the SGN (Fig. 4A) (Kimura et al., 2005; Ghimire et al., 2018). The Emx2-Cre driver effectively deletes the HA-tagged, floxed Fzd3 allele based upon the reduction in Fzd3 ISH signaling in the organ of Corti (Fig. $4 B, C)$ and loss of FZD3-HA immunofluorescence from the boundaries between neighboring supporting cells (Fig. 4D,E). While Emx2-Cre;Fzd3 CKO; Fzd6 KO cochlea contain a comparable number of Type II SGNs, on average 55\% of mutant fibers turn incorrectly toward the cochlear apex while turning errors are negligible in littermate controls (Fig. $4 F-H$ ). Together, these phenotype analyses demonstrate that Fzd3/Fzd6 genetic redundancy occurs in the organ of Corti and that FZD3 and FZD6 contribute to axon guidance through a non-cell-autonomous mechanism. This spatially restricted and non-cell-autonomous phenotype resembles prior Vangl2 CKO analyses (Ghimire et al., 2018) and demonstrates that a PCP signaling complex formed between organ of Corti-supporting cells guides peripheral axon turning.

Frizzled 3 and Vangl2 function in a common pathway The similarity of phenotypes between the Vangl2 KO, CELSR1 $\mathrm{KO}$, and Fzd3;Fzd6 dKOs, and the distribution of these core PCP proteins at the boundaries between neighboring supporting cells suggest that peripheral axon turning is guided by an intercellular protein complex comprised of these factors. In other systems, the formation of intercellular PCP complexes requires the asymmetric distribution of each PCP protein, and protein distribution is lost when individual PCP proteins are removed (Goodrich and Strutt, 2011). This functional relationship was tested by immunofluorescent labeling of VANGL2 and CELSR1 proteins in $F z d 3 / 6$ dKOs. In the absence of Fzd3 and Fzd6, VANGL2 and CELSR1 are retained at the apical surface of the cochlear duct; however, their asymmetric distribution is lost, and they frequently surround individual cells (Fig. 5). This occurs in the or- 


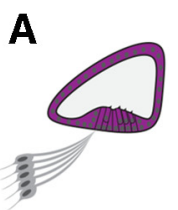

Emx2-Cre; Rosa tdTomato
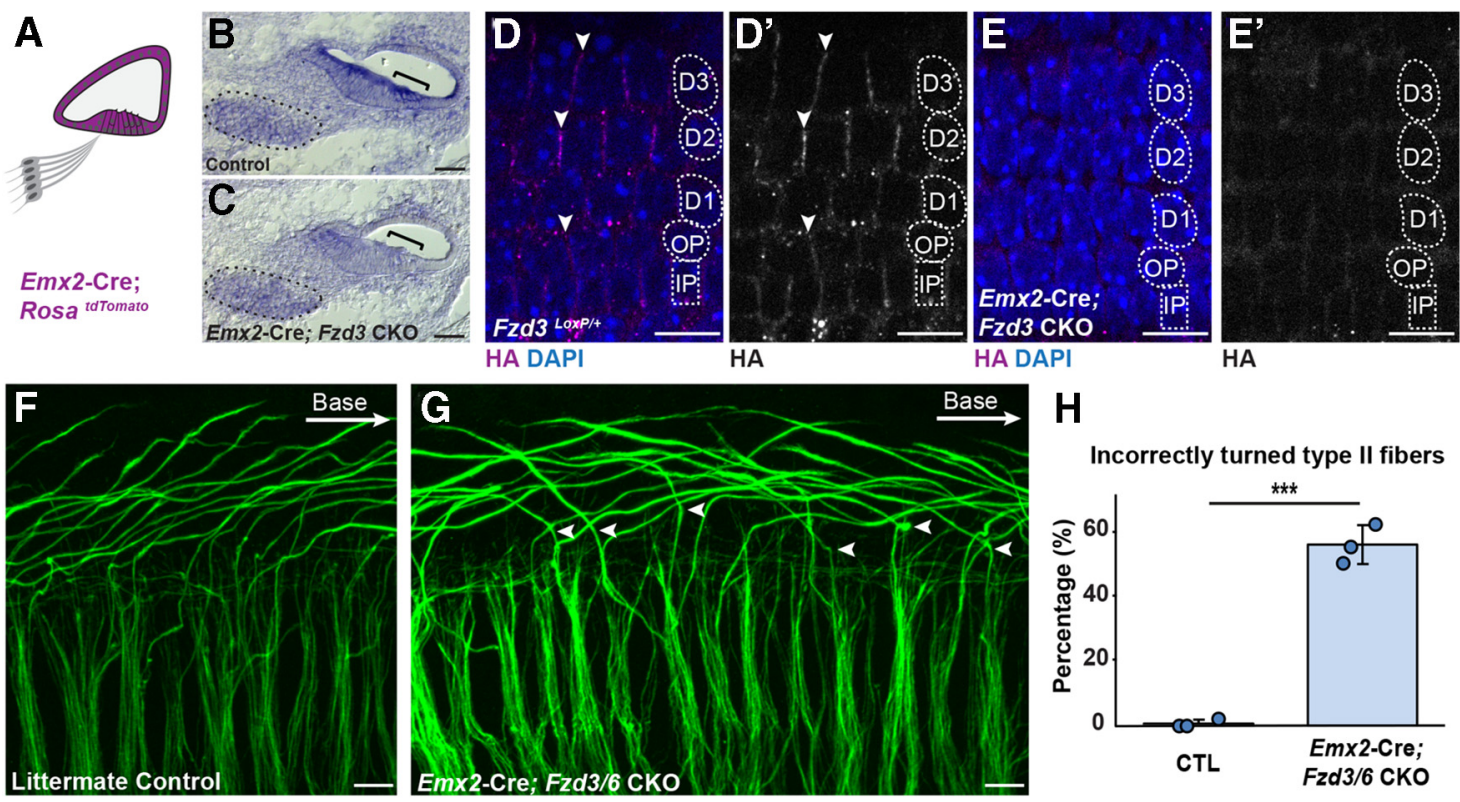

$\mathrm{H}$

Incorrectly turned type II fibers

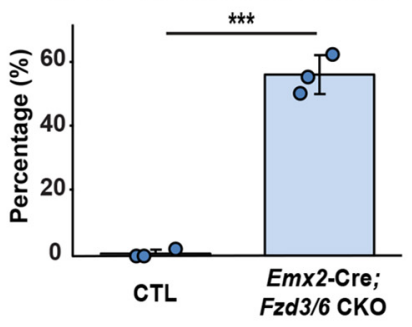

Figure 4. Type II turning is regulated by nonautonomous Fzd3/Fzd6 function. $\boldsymbol{A}$, Schematic diagram showing the pattern of Emx2-Cre-mediated recombination in the cochlear duct. $\boldsymbol{B}, \boldsymbol{C}$, Conventional ISH showing that the reduction of Fzd3 mRNA at E16.5 in the organ of Corti (bracket) but not the SGN (outlined) from Emx2-Cre; Fzd3 CKOs. D, D', E, E', FZD3-HA expression is also lost from the basolateral junctions between neighboring supporting cells in Emx2-Cre; Fzd3 CKOs compared with littermate controls. $F, G, N F 200$ immunolabeling of Type II SGN peripheral axons in Emx2-Cre;Fzd3 CK0;Fzd6 KOs (Emx2-Cre;Fzd3/6 CKO) reveals multiple turning errors (arrowheads) compared with littermate controls in the basal turn of the E18.5 cochlea. $\boldsymbol{H}$, Average frequency of incorrectly turned Type ll fibers projecting toward the cochlear apex. Data are mean \pm SD with overlying circles reporting to the frequency of turning errors for individual mice. A single cochlea was analyzed from each animal: Control ( $n=3,127$ axons), CKOs ( $n=3,142$ axons). ${ }^{* * *} p \leq 0.001$, CKOs versus littermate controls (Student's $t$ test). Supporting cell nuclei are identified based upon DAPI staining of nuclei: IPCs, OPCs, first (D1), second (D2), and third (D3) row Deiters' cells. Scale bars, $10 \mu \mathrm{m}$.

gan of Corti, where PCP proteins are distributed at cell junctions oriented parallel to the long axis of the cochlea; and in nonsensory cells of Kollicker's organ, where PCP proteins are distributed at cell junctions oriented parallel to the mediolateral axis (Fig. $5 A-D)$. Similarly, along the basolateral edges of cochlearsupporting cells, the asymmetric distribution of VANGL2 and CELSR1 is lost in the absence of $F z d 3$ and Fzd6 (Fig. 5E-H). Together, these protein distribution assays show that FZD3, FZD6, VANGL2, and CELSR1 form an interdependent protein complex between organ of Corti-supporting cells.

The potential for these proteins to act within a common signaling complex was evaluated through a series of genetic interaction studies. In Vangl2 CKOs generated using Pax2-Cre; 35\% of fibers turn incorrectly toward the cochlear apex (Fig. 6B, G,H). The penetrance of the phenotype is less than Vangl2 KOs where turning is randomized with $50 \%$ of fibers turning incorrectly (Ghimire et al., 2018). While this difference is likely due to perdurance of the VANGL2 protein or mRNA following Cremediated gene deletion, the decreased penetrance of the phenotype in Pax2-Cre; Vangl2 CKOs allows genetic interactions between Vangl2 and Fzd3 to be tested by phenotypic enhancement. For these assays, peripheral axons were evaluated at E18.5 in the basal turn. When Pax2-Cre;Vangl2 CKO axons were evaluated on a $F z d 3 \mathrm{KO}$ background, the frequency of turning errors increased to 50\%, consistent with the hypothesis that Vangl2 and $\mathrm{Fzd} 3$ act in a common protein complex and signaling pathway (Fig. $6 D, G$ ). A similar genetic interaction assay was conducted for the noncanonical WNT-receptor ROR2 which, such as Fzd3 and Fzd6, has been linked to stereociliary bundle PCP in hair cells (Yamamoto et al., 2008). Despite this, no turning errors were identified in Pax2-Cre;ROR2 CKOs (Fig. 6E), and the penetrance of the Pax2-Cre; Vangl2 CKO phenotype was not enhanced with the concomitant loss of ROR2 (Fig. 6F,H). Together, these ge- netic interaction assays indicate that $F z d 3$ and $F z d 6$ encode the principle WNT receptors cooperating with VANGL2 to guide peripheral axon turning.

\section{WNT signaling is required to pattern the cochlear sensory epithelia}

It has been proposed that the asymmetric distribution of $\mathrm{PCP}$ proteins is established by a noncanonical WNT gradient and is subsequently maintained or propagated between neighbors by the PCP signaling complex (Yang and Mlodzik, 2015). To test this hypothesis in the cochlea, Wnt5a KO cochlea were evaluated at E18.5 for Type II peripheral axon turning defects. WNT5a is a noncanonical WNT that has been linked to cochlear hair cell polarization (Qian et al., 2007) as well as axon guidance in the CNS (Fenstermaker et al., 2010; Morello et al., 2015). Despite these precedents, Type II fiber turning appears normal in Wnt5a KOs and is comparable with littermate controls (Fig.7 A, B,K). The mouse genome contains 19 Wnt genes so the likelihood of genetic compensation for the loss of Wnt5a is high. However, all WNT proteins are processed and secreted through a trafficking pathway that is dependent upon the porcupine O-acyltransferase, and WNT secretion can be disrupted by targeting the Porcn gene encoding this acyltransferase (Barrott et al., 2011). Consistent with this, Pax2-Cre;Porcn CKOs have smaller temporal bones and shorter cochlea compared with littermate controls (Fig. 7C-F). However, WNT depletion is not complete in this mutant, and the gross phenotype is likely hypomorphic because canonical WNT signaling is also required for hair cell differentiation (Shi et al., 2010), yet hair cells are retained throughout the Pax2-Cre;Porcn CKO cochlea (Fig. 7G,H). Nonetheless, evaluation of Type II fibers in Pax2-Cre;Porcn CKOs showed incorrectly turned fibers (Fig. $7 I, J, L$, blue arrowheads). In addition, fibers that appear stalled or branched are more frequent in Pax2-Cre;PORCN 

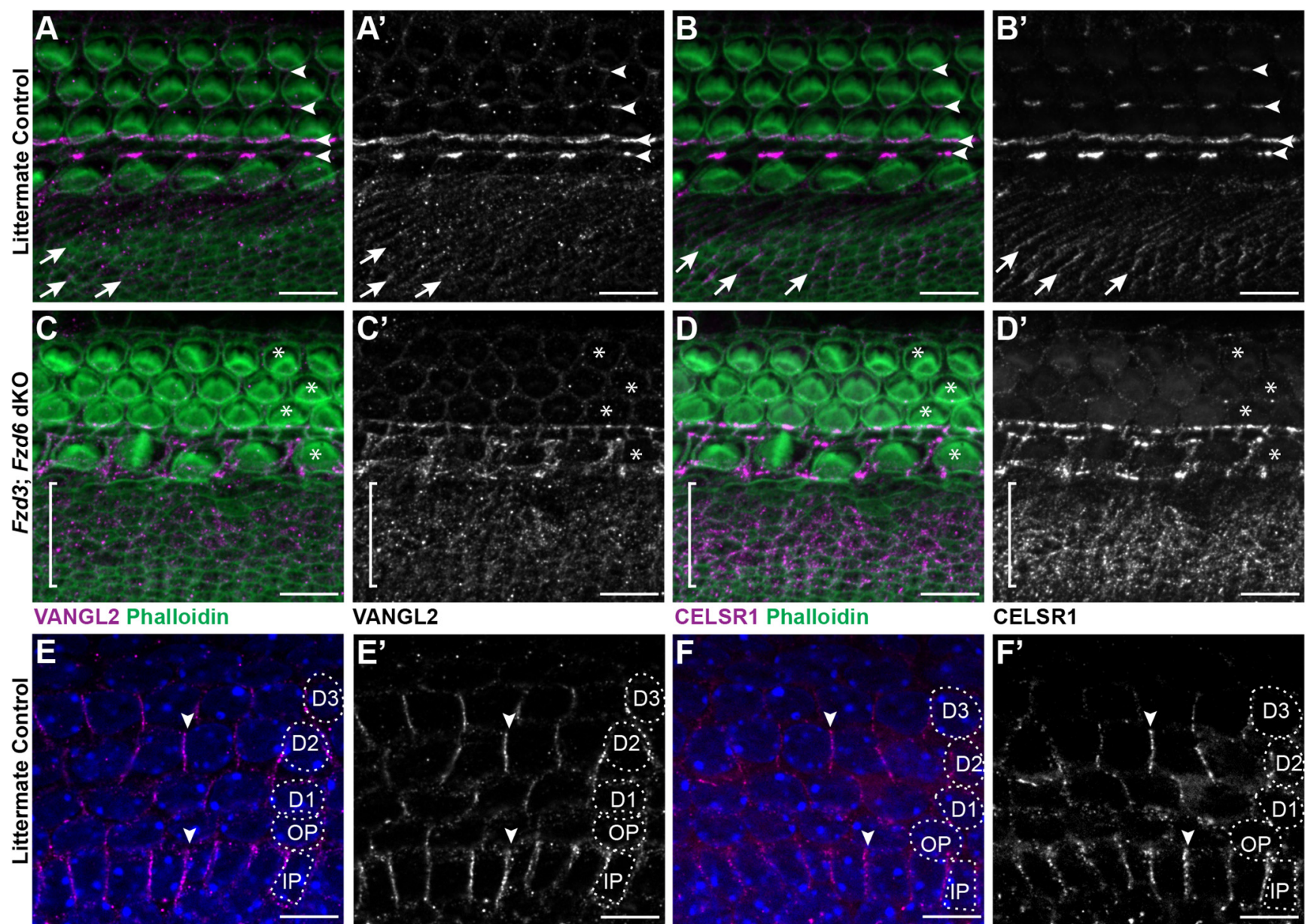

\section{VANGL2}

CELSR1 Phalloidin

CELSR1
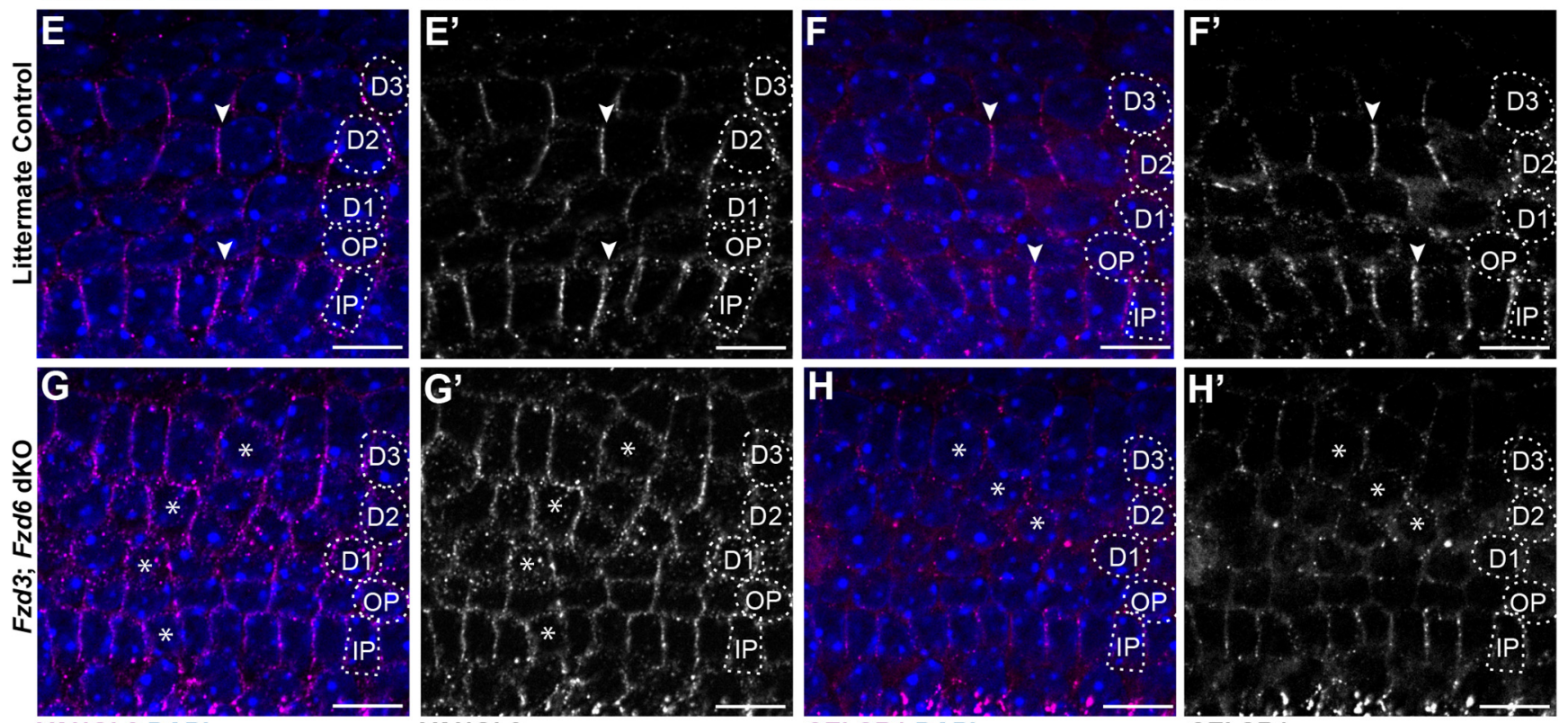

VANGL2

CELSR1 DAPI

CELSR1

Figure 5. Loss of $F z d 3$ and $F z d 6$ disrupts the polarized distribution of the remaining core PCP proteins. $A, \boldsymbol{A}^{\prime}, \boldsymbol{B}, \boldsymbol{B}^{\prime}$, Immunofluorescent labeling of VANGL2 or CELSR1 (magenta) on the apical surface of the organ of Corti shows the asymmetric distribution of these proteins at hair cell to supporting cell junctions (arrowheads), and between nonsensory cell boundaries (arrows) in the greater epithelial ridge of littermate controls. C, $\mathbf{C}^{\prime}, \mathbf{D}, \mathbf{D}^{\prime}$, In Fzd3; Fzd6 dKOs, VANGL2 and CELSR1 distribution is disrupted around hair cells (examples marked by asterisk) and throughout the GER region (bracket). Moreover, the localization of VANGL2 and CELSR1 appear to be completely lost in the $0 H$ C region. $\boldsymbol{E}_{,} \boldsymbol{E}^{\prime}, \boldsymbol{F}, \boldsymbol{F}^{\prime}$, The asymmetric distribution of VANGL2 and CELSR1 at basolateral membrane of supporting cells in control tissue resembles FZD distribution at this location. $\mathbf{G}, \mathbf{G}^{\prime}, \boldsymbol{H}_{,} \boldsymbol{H}^{\prime}$, In Fzd3; Fzd6 dKOs, VANGL2 and CELSR1 levels appear reduced and the proteins encircle many supporting cells. All images are from E18.5 cochlea. Supporting cell nuclei are identified based upon DAPI staining of nuclei: IPCs, OPCs, first (D1), second (D2), and third (D3) row Deiters' cells. Scale bars, $10 \mu \mathrm{m}$.

CKOs than littermate controls (Fig. 7J,L, orange arrowheads), and mutant fibers with these morphologies have not been seen in PCP mutants. The asymmetric distribution of core PCP proteins was also disrupted in Pax2-Cre;Porcn CKOs consistent with the hypothesis that noncanonical WNTs initiate PCP protein distribution. Thus VANGL2 and CELSR1 are altered in Pax2-Cre; Porcn CKOs and frequently appear to surround individual supporting cells (Fig. 8) in a pattern of redistribution that resembles those changes that occur in Fzd3;Fzd6 dKOs (Fig. 5). Although all members of the PCP complex were present, their highly patterned organization along the basolateral surfaces of supporting cells was lost. Since the core PCP proteins function non-cell-autonomously from cochlear-supporting cells to guide Type II peripheral axon turning, these changes in protein distribution likely underlie the turning errors and stalling observed in Pax2-Cre;Porcn CKOs.

\section{Discussion}

Using a combination of $\mathrm{KO}$ and conditional $\mathrm{KO}$ mice, we demonstrate a WNT-dependent intercellular PCP signaling complex between cochlear-supporting cells that contributes to the guidance event directing Type II SGN peripheral axons to turn toward the cochlear base. The noncanonical WNT receptors FZD3 and FZD6 are functionally redundant in this context, similar to their 

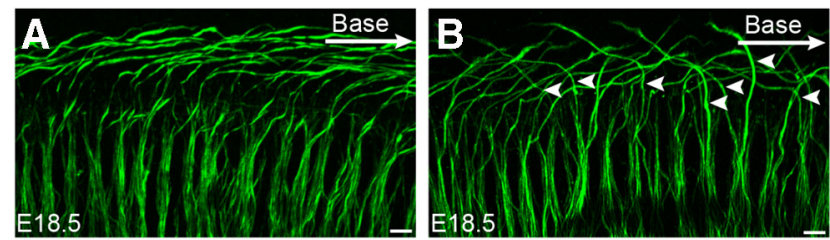

\section{Littermate Control} Pax2-Cre; Vangl2 CKO

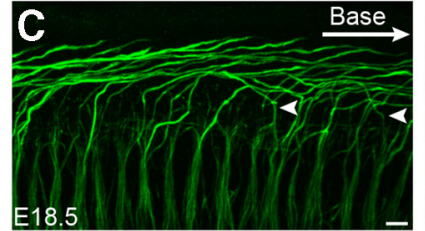

Fzd3 KO

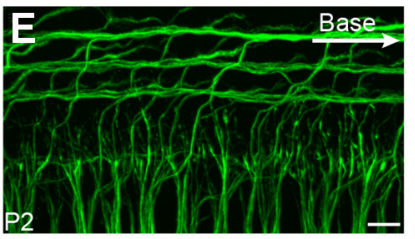

Pax2-Cre; ROR2 CKO

\section{G Incorrectly turned} type II fibers

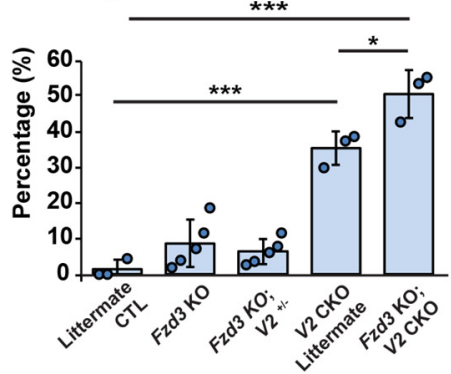

H Incorrectly turned type II fibers

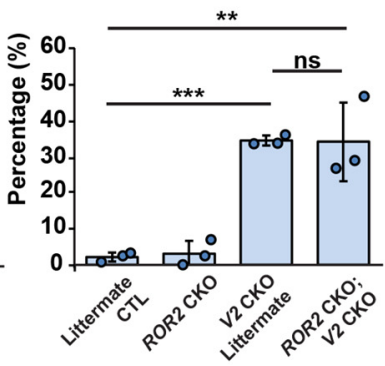

Figure 6. Fzd3, and not ROR2, genetically interacts with Vangl2 in SGN development. A-D, NF200 immunolabeling of Type II SGN peripheral axons in Pax2-Cre; Vangl2 CKOs and littermate controls generated on a Fzd3 $\mathrm{KO}$ background. Arrowheads indicate Type II fibers, which have turned incorrectly toward the cochlear apex. E, F, NF200 labeled Type II peripheral axons in Pax2-Cre;ROR2 CKO and Pax2-Cre;ROR2; VangI2 double CKOs. G, Quantification of the average frequency of turning errors for cochlea of each genotype demonstrates a genetic enhancement with the loss of Vangl2 and Fzd3. H, Similar quantification of turning errors in Pax2-Cre; ROR1; Vangl2 double CKOs did not reveal a genetic interaction between Vangl2 and ROR2. Data are mean $\pm S D$ with overlying circles reporting to the frequency of turning errors for individual mice. A single cochlea was analyzed from each animal. For Vangl2-Fzd3 interaction assays, images and quantification are from the basal turn of E18.5 cochlea: Control ( $n=3,159$ axons), Fzd3 KO ( $n=5,248$ axons), Fzd3 KO, Vangl2 ${ }^{+1-}(n=5,234$ axons), Vangl2 CKO ( $n=3,101$ axons), Fzd3 K0; Vangl2 CKO ( $n=3,117$ axons). For Vangl2-ROR2 interaction assays, images are from the basal turn, and quantification is summed from basal, middle, and apical turns: Control ( $n=3,375$ axons), ROR2 CKO ( $n=3,380$ axons), Vangl2 CKO ( $n=3,324$ axons), ROR2 CKO; Vangl2 CKO ( $n=3,313$ axons). ${ }^{*} p \leq 0.05 ;{ }^{* *} p \leq 0.01 ;{ }^{* *} p \leq 0.001$; ns (not significant); pairwise comparisons as indicated (Student's $t$ test). All images are from E18.5 cochlea and scalebars are $10 \mu \mathrm{m}$.

function in hair cell PCP (Wang et al., 2006), and growth cone turning is randomized when Fzd3 and Fzd6 are deleted (Fig. 3). We further demonstrate that the site of this functional redundancy is within the cochlear duct (Fig. 4), similar to VANGL2 (Ghimire et al., 2018) and revealing that FZD3 and FZD6 also function through a non-cell-autonomous mechanism. Since FZD3, FZD6, and VANGL2 are core elements of the noncanonical Wnt-PCP signaling complex and interact genetically in the cochlea (Fig. 6), we propose that a planar polarity axis established in the cochlear duct guides cochlear innervation. This mechanism differs from that guiding commissural axons of the neural
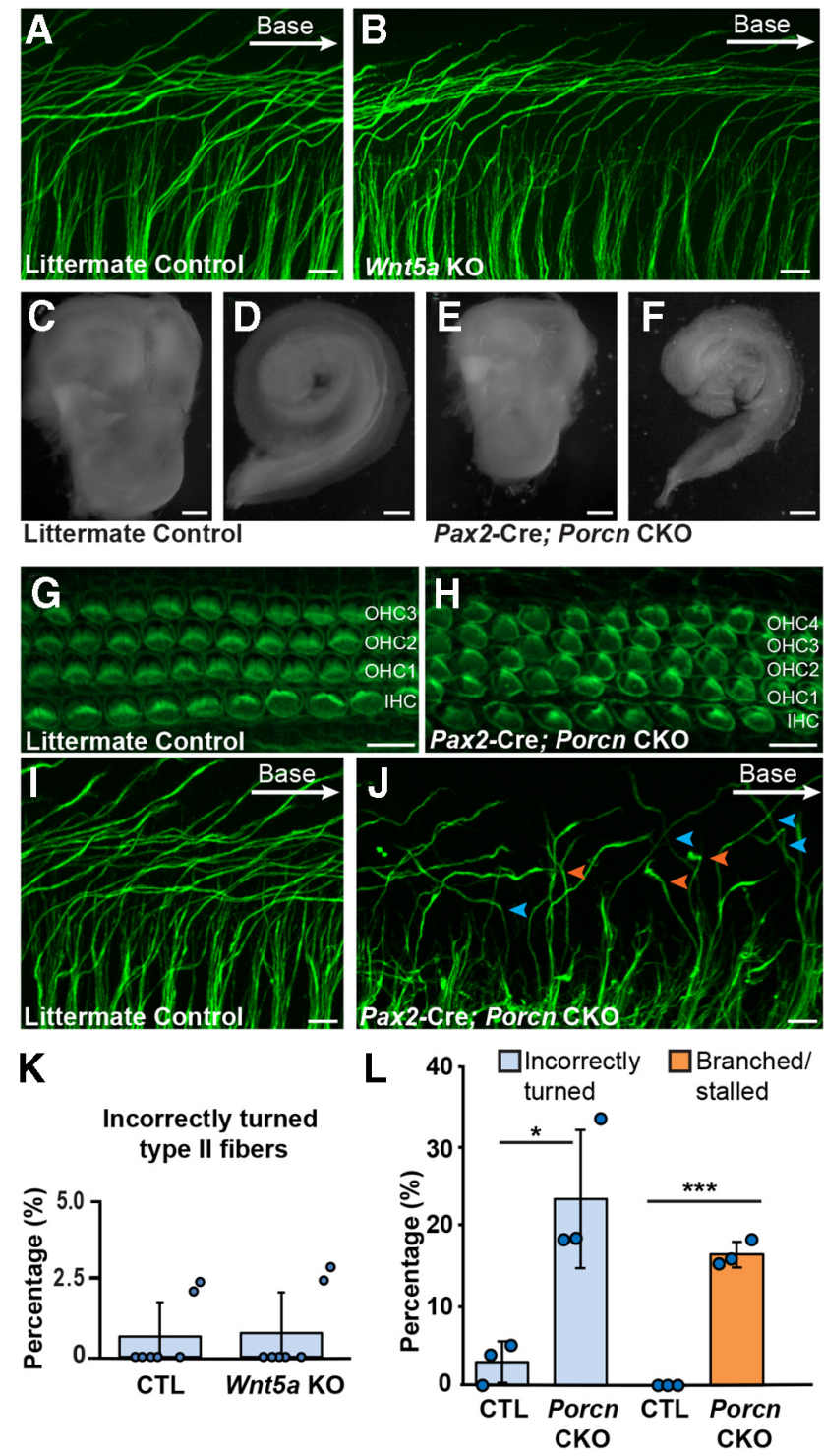

Figure 7. WNT activity is required for Type II SGN axon guidance. $\boldsymbol{A}, \boldsymbol{B}, \mathrm{NF} 200 \mathrm{imm}$ unolabeling of Type II SGN peripheral axons in Wnt5a KO and littermate controls reveals no turning errors in the basal turn at E18.5. C-F, Modest impairment of inner ear development in Pax2-Cre;Porcn CKOs compared with littermate controls revealed by smaller temporal bones $(\boldsymbol{C}, \boldsymbol{E})$ and cochlea $(\boldsymbol{D}, \boldsymbol{F})$ in CKOs. $\boldsymbol{G}, \boldsymbol{H}$, Phalloidin staining of stereociliary bundles demonstrates hair cell differentiation in Pax2-Cre;Porcn CKOs in addition to an extra row of $\mathrm{OHCS}(\mathrm{OHC}$ ) throughout the length of the cochlea. I, J, Type II peripheral axons are more likely to turn incorrectly (blue arrowheads) toward the cochlear apex in Porcn CKOs compared with littermate control, and stalled or branched fibers are also prevalent (orange arrowheads). $\boldsymbol{K}$, $\boldsymbol{L}$, Quantification of innervation defects in WNT signaling mutants their respective littermate controls. Data are mean \pm SD with overlying circles reporting to the frequency of turning errors for individual mice. All images and quantification were collected from the basal turn of E18.5 cochlea, with a single cochlea analyzed from each animal: Control ( $n=7,312$ axons), Wnt5a KO ( $n=7,308$ axons), Porcn controls ( $n=3,139$ axons), Porcn CKOs ( $n=3,115$ axons). IHCs, OHCs (OHC1, OHC2, OHC3), and an extra row $\mathrm{OHCs}(\mathrm{OHC}())^{*} p \leq 0.05 ;{ }^{* * *} p \leq 0.001$; mutants versus littermate controls (Student's $t$ test). Scale bars, $10 \mu \mathrm{m}$.

tube where PCP proteins function autonomously from within the growth cone to promote turning in response to a Wnt gradient (Zou, 2004, 2012). Despite this difference, Type II SGN turning also remains WNT-dependent because blocking Wnt release through the conditional deletion of the Porcn gene results in similar axon turning errors (Fig. 8).

Based upon these observations and our prior phenotypic analyses of Vangl2 mutants (Ghimire et al., 2018), we propose a 

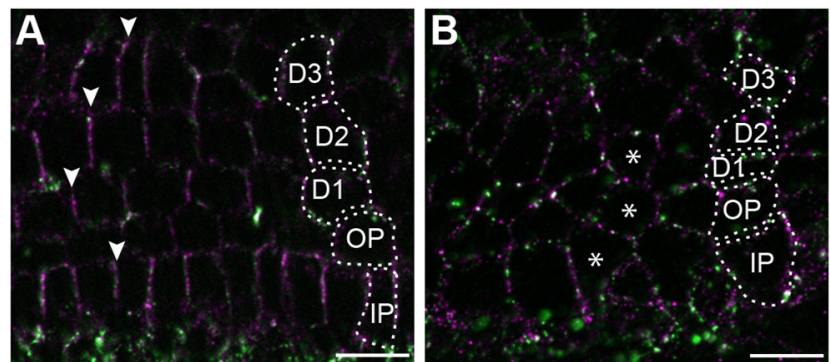

\section{Control}

VANGL2 CELSR1 Porcn CKO

VANGL2 CELSR1
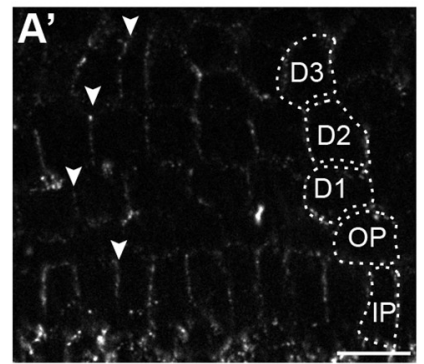

CELSR1

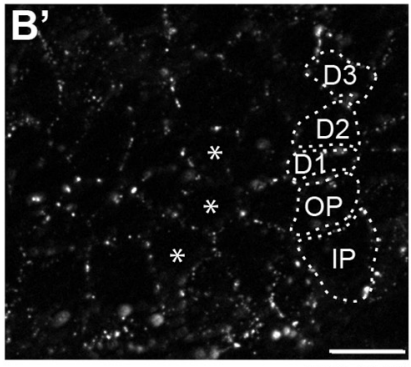

CELSR1
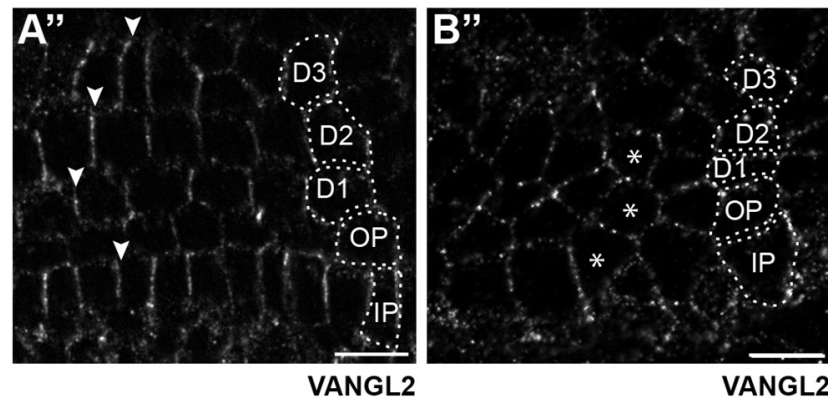

VANGL2

VANGL2

Figure 8. WNT mediated signaling establishes PCP axis in cochlear-supporting cells. The asymmetric distribution of VANGL2 and CELSR1 along the basolateral boundaries of cochlearsupporting cells of littermate controls $\left(\boldsymbol{A}-\boldsymbol{A}^{\prime \prime}\right)$ is disrupted in Pax2-Cre;Porcn CKOs $\left(\boldsymbol{B}-\boldsymbol{B}^{\prime \prime}\right)$. Arrowheads indicate examples of asymmetric protein distribution in controls. Asterisks indicate examples of supporting cells that lack asymmetric protein distributions in CKOs. Supporting cell nuclei are identified based upon DAPI staining of nuclei; IPCS, OPCS, first (D1), second (D2), and third (D3) row Deiters' cells. Scale bars, $10 \mu \mathrm{m}$.

model in which noncanonical Wnt-PCP signaling establishes a planar polarity axis relayed between neighboring supporting cells of the cochlear duct (Fig. 9A). In this model, PCP signaling results in the polarized distribution of protein arrays consisting of the core PCP proteins and potentially additional factors, such as axon guidance cues, that provide directional information to the incoming growth cone (Fig. 9B). FZD3/6 and VANGL2 could function non-cell-autonomously in this context in one of two ways. First, the intercellular PCP signal relayed between supporting cells by FZD3/6 and VANGL2 could be interpreted by the growth cone if it contains complementary PCP proteins. For example, an FZD receptor in the growth cone could detect the asymmetric distribution of VANGL2 in supporting cells. Second, the planar polarization of supporting cells could direct the subcellular distribution of membrane-tethered axon guidance cues, such as semaphorins or ephrins. In this scenario, a repulsive guidance cue restricted to the side of supporting cells facing the cochlear base could theoretically promote growth cone turning, although this repulsive force must also not be so strong as to prevent the growth cone from entering the intercellular space. Consistent with this hypothesis, PCP signaling occurs upstream of Eph/ephrin signaling in the developing hindlimb (Chai et al., 2014), although whether this involves the regulated distribution of Ephrin

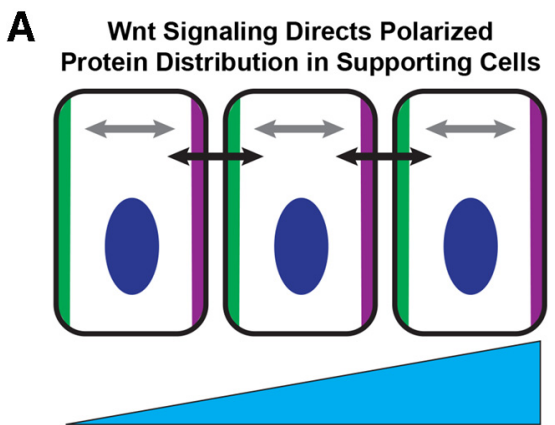

B

Polarized Protein Arrays Direct Growth Cone Turning Towards the Base

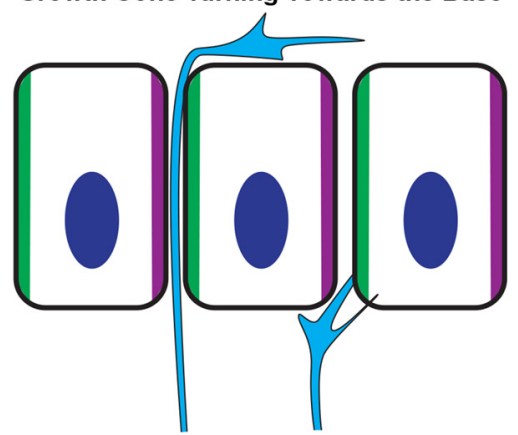

Figure 9. Planar polarity of cochlear-supporting cells directs peripheral axon turning. $\boldsymbol{A}$, WNT signaling, perhaps acting in a gradient, promotes the asymmetric distribution of the core $P C P$ proteins (green and magenta) within organ of Corti-supporting cells. This distribution is reinforced by intracellular feedback loops within individual cells (gray arrows) and intercellular signaling between neighboring cells (black arrows). This planar polarization mechanism may also direct the polarized subcellular distribution of membrane tethered axon guidance molecules, such as ephrins or semaphorins. $\boldsymbol{B}$, As the growth cone of the peripheral axon enters this polarized environment, it is directed toward the cochlear base by the polarized array of proteins that it encounters before turning. Growth cone turning could occur in response to intercellular PCP signaling between supporting cells and the growth cone, or to the polarized distribution of axon guidance cues that are asymmetrically distributed through a PCP-dependent mechanism.

ligands is not known. While our phenotypic analyses do not distinguish between these alternatives, they do demonstrate that FZD3/FZD6 have a non-cell-autonomous function in cochlearsupporting cells similar to VANGL2 (Ghimire et al., 2018).

Inner ear hair cells have emerged as a model for vertebrate PCP signaling, with stereociliary bundle orientation dependent upon orthologous genes to those regulating PCP in Drosophila (Wang and Nathans, 2007; Goodrich and Strutt, 2011), and vertebrate PCP proteins signaling between hair cells and supporting cells to coordinate cellular polarity (Sienknecht et al., 2011; Stoller et al., 2016). Despite this well-documented conservation of PCP genes in hair cell development, hair cell planar polarity does not influence or direct peripheral axon turning. Foremost, the Type II SGN peripheral axon turns in the vicinity of the basolateral walls of cochlear-supporting cells and before contacting OHCs (Fig. 1) (Ghimire et al., 2018). Furthermore, turning errors are prevalent in the CELSR1 KO cochlea in which IHC and $\mathrm{OHC}$ stereociliary bundle orientation is normal (Duncan et al., 2017). Last, selective deletion of Vangl2 from supporting cells using FGFR3-iCreER is sufficient to induce a high frequency of turning errors (Ghimire et al., 2018). Consistent with these findings, the core PCP proteins are asymmetrically distributed at the basolateral intercellular junctions between neighboring supporting cells. FZD3 is enriched along one side of supporting cells facing the cochlear base (Fig. 2) and apposes VANGL2 localized along the side of supporting cells facing the cochlear apex (Ghi- 
mire et al., 2018). Similar to the interdependent distribution of PCP proteins in other systems, Fzd3 and Fzd6 deletion disrupts the polarized distributions of VANGL2 and CELSR1 (Fig. 5). Thus, an intercellular PCP signaling complex composed of FZD3, FZD6, CELSR1, and VANGL2 is present at the site of growth cone turning. Remarkably, while a similar intercellular PCP complex at the apical surface of the organ of Corti guides stereociliary bundle orientation, these PCP proteins are enriched at cell boundaries oriented parallel to the long axis of the cochlea while basolateral PCP proteins are enriched at boundaries perpendicular to this axis. As a result, the cochlea is patterned by two independent and functionally distinct PCP axes that are maintained by a common cohort of IPCs and OPCs and Deiters' cells. An outstanding question is how these cells become polarized during development, and are able to maintain these asymmetric protein localizations in separate subcellular compartments to sustain these independent PCP axes.

In the developing neural tube, axon outgrowth follows an increasing gradient of WNT activity. In the mouse, Wnt4 is expressed in an anterior to posterior gradient that promotes growth cone turning and caudad extension of commissural axons (Lyuksyutova et al., 2003). A similar gradient of WNT activity is established in the chick through a posterior to anterior gradient of the WNT inhibitor SFRP that sculpts a complementary gradient of Wnt activity (Domanitskaya et al., 2010). Protein localization and trafficking studies implicate core PCP proteins in the growth cone as interpreting this gradient (Onishi et al., 2013; Onishi and Zou, 2017). The data that we present here suggest that, unlike the spinal cord, WNT does not act as a diffusible axon guidance cue in the developing cochlea. Analysis of Porcn CKOs further suggests that the contribution of WNT signaling may be to establish the asymmetric distributions of FZD3, FZD6, and VANGL2 at the basolateral junctions between cochlearsupporting cells rather than as a diffusible attractant. While this study does not identify the specific WNT ligand contributing to these events, it does exclude the necessity of WNT5a. One possibility is that WNT7a, another noncanonical Wnt associated with inner ear PCP (Dabdoub et al., 2003), regulates these events in conjunction with, or as an alternative to, WNT5a.

The orderly array of hair cells and supporting cells is also disrupted when WNT signaling is compromised in the Porcn $\mathrm{CKO}$ cochlea. This raises the possibility that the asymmetric distributions of VANGL2 and CELSR1 are more dependent upon the organization of cells and cell boundaries than WNT gradients. Our analyses do not dismiss this possibility, and it remains consistent with our model in which the distribution of PCP proteins in the environment is essential for directing growth cone turning toward the cochlear base. However, disrupted cellular patterning is unlikely to be the basis of turning errors in other PCP mutants because the organization of hair cells and supporting cells is not altered in Fzd3/6 dKOs. Furthermore, the occurrence of Type II SGN turning errors is not restricted to PCP mutants, and similar phenotypes have been described following the ablation of the transcription factor Proxl (Fritzsch et al., 2010) and the canonical WNT signaling regulator gene Rspo2 (Mulvaney et al., 2013). An interesting hypothesis is that PROX1 could directly regulate peripheral axon guidance by controlling the expression of core PCP proteins; however, this possibility has yet to be tested. In contrast, the basis of the Rspo2 phenotype may be less direct and more similar to the Porcn CKO phenotype because cellular patterning is disrupted in Rspo2 mutants. As a result, the polarized organization of PCP proteins along the trajectory of the migrating growth cone may also be altered.
This PCP-dependent mechanism for guiding peripheral axon turning provides several advantages for the developing cochlea. First, it has the potential to self-assemble and maintain itself due to the intercellular interaction of PCP proteins. Thus, an early and transient WNT signal could establish the PCP axis and would not have to be maintained as the cochlear duct elongates and spirals. Second, this mechanism would be resilient to potential disruptions from the dynamic cellular rearrangements that occur during cochlear duct outgrowth similar to the PCP axes that are maintained throughout gastrulation and convergent extension of the neural tube. Third, this guidance mechanism is modular, and turning signals with comparable magnitude and distribution are presented to every individual growth cone along the entire length of the cochlea. As a result, the remarkable fidelity of Type II SGN peripheral axon turning can be maintained throughout the full extent of the cochlear spiral. Future studies are needed to determine whether this is a developmental mechanism that has evolved specifically within the cochlea or whether these principles contribute to axon guidance for other populations of neurons in the CNS.

\section{References}

Barrott JJ, Cash GM, Smith AP, Barrow JR, Murtaugh LC (2011) Deletion of mouse Porcn blocks Wnt ligand secretion and reveals an ectodermal etiology of human focal dermal hypoplasia/Goltz syndrome. Proc Natl Acad Sci U S A 108:12752-12757.

Chai G, Zhou L, Manto M, Helmbacher F, Clotman F, Goffinet AM, Tissir F (2014) Celsr3 is required in motor neurons to steer their axons in the hindlimb. Nat Neurosci 17:1171-1179.

Copley CO, Duncan JS, Liu C, Cheng H, Deans MR (2013) Postnatal refinement of auditory hair cell planar polarity deficits occurs in the absence of Vangl2. J Neurosci 33:14001-14016.

Curtin JA, Quint E, Tsipouri V, Arkell RM, Cattanach B, Copp AJ, Henderson DJ, Spurr N, Stanier P, Fisher EM, Nolan PM, Steel KP, Brown SD, Gray IC, Murdoch JN (2003) Mutation of Celsrl disrupts planar polarity of inner ear hair cells and causes severe neural tube defects in the mouse. Curr Biol 13:1129-1133.

Dabdoub A, Donohue MJ, Brennan A, Wolf V, Montcouquiol M, Sassoon DA, Hseih JC, Rubin JS, Salinas PC, Kelley MW (2003) Wnt signaling mediates reorientation of outer hair cell stereociliary bundles in the mammalian cochlea. Development 130:2375-2384.

Davey CF, Mathewson AW, Moens CB (2016) PCP signaling between migrating neurons and their planar-polarized neuroepithelial environment controls filopodial dynamics and directional migration. PLoS Genet 12: e1005934.

Devenport D, Fuchs E (2008) Planar polarization in embryonic epidermis orchestrates global asymmetric morphogenesis of hair follicles. Nature cell biology 10:1257-1268.

Domanitskaya E, Wacker A, Mauti O, Baeriswyl T, Esteve P, Bovolenta P, Stoeckli ET (2010) Sonic hedgehog guides post-crossing commissural axons both directly and indirectly by regulating Wnt activity. J Neurosci 30:11167-11176.

Duncan JS, Stoller ML, Francl AF, Tissir F, Devenport D, Deans MR (2017) Celsr 1 coordinates the planar polarity of vestibular hair cells during inner ear development. Dev Biol 423:126-137.

Feng J, Xian Q, Guan T, Hu J, Wang M, Huang Y, So KF, Evans SM, Chai G, Goffinet AM, Qu Y, Zhou L (2016) Celsr3 and Fzd3 organize a pioneer neuron scaffold to steer growing thalamocortical axons. Cereb Cortex 26:3323-3334.

Fenstermaker AG, Prasad AA, Bechara A, Adolfs Y, Tissir F, Goffinet A, Zou Y, Pasterkamp RJ (2010) Wnt/planar cell polarity signaling controls the anterior-posterior organization of monoaminergic axons in the brainstem. J Neurosci 30:16053-16064.

Flores EN, Duggan A, Madathany T, Hogan AK, Márquez FG, Kumar G, Seal RP, Edwards RH, Liberman MC, García-Añoveros J (2015) A noncanonical pathway from cochlea to brain signals tissue-damaging noise. Curr Biol 25:606-612.

Fritzsch B, Dillard M, Lavado A, Harvey NL, Jahan I (2010) Canal cristae growth and fiber extension to the outer hair cells of the mouse ear require Prox1 activity. PLoS One 5:e9377. 
Ghimire SR, Ratzan EM, Deans MR (2018) A non-autonomous function of the core PCP protein VANGL2 directs peripheral axon turning in the developing cochlea. Development 145:dev159012.

Giese AP, Ezan J, Wang L, Lasvaux L, Lembo F, Mazzocco C, Richard E, Reboul J, Borg JP, Kelley MW, Sans N, Brigande J, Montcouquiol M (2012) Gipcl has a dual role in Vangl2 trafficking and hair bundle integrity in the inner ear. Development 139:3775-3785.

Glasco DM, Sittaramane V, Bryant W, Fritzsch B, Sawant A, Paudyal A, Stewart M, Andre P, Cadete Vilhais-Neto G, Yang Y, Song MR, Murdoch JN, Chandrasekhar A (2012) The mouse Wnt/PCP protein Vangl2 is necessary for migration of facial branchiomotor neurons, and functions independently of dishevelled. Dev Biol 369:211-222.

Goodrich LV, Strutt D (2011) Principles of planar polarity in animal development. Development 138:1877-1892.

Guo N, Hawkins C, Nathans J (2004) Frizzled6 controls hair patterning in mice. Proc Natl Acad Sci U S A 101:9277-9281.

Ho HY, Susman MW, Bikoff JB, Ryu YK, Jonas AM, Hu L, Kuruvilla R, Greenberg ME (2012) Wnt5a-ror-dishevelled signaling constitutes a core developmental pathway that controls tissue morphogenesis. Proc Natl Acad Sci U S A 109:4044-4051.

Hua ZL, Smallwood PM, Nathans J (2013) Frizzled3 controls axonal development in distinct populations of cranial and spinal motor neurons. Elife 2:e01482.

Hua ZL, Jeon S, Caterina MJ, Nathans J (2014a) Frizzled3 is required for the development of multiple axon tracts in the mouse central nervous system. Proc Natl Acad Sci U S A 111:E3005-E3014.

Hua ZL, Chang H, Wang Y, Smallwood PM, Nathans J (2014b) Partial interchangeability of Fz3 and Fz6 in tissue polarity signaling for epithelial orientation and axon growth and guidance. Development 141:39443954.

Kimura J, Suda Y, Kurokawa D, Hossain ZM, Nakamura M, Takahashi M, Hara A, Aizawa S (2005) Emx2 and Pax6 function in cooperation with Otx2 and Otx1 to develop caudal forebrain primordium that includes future archipallium. J Neurosci 25:5097-5108.

Liu C, Glowatzki E, Fuchs PA (2015) Unmyelinated Type II afferent neurons report cochlear damage. Proc Natl Acad Sci U S A 112:14723-14727.

Lyuksyutova AI, Lu CC, Milanesio N, King LA, Guo N, Wang Y, Nathans J, Tessier-Lavigne M, Zou Y (2003) Anterior-posterior guidance of commissural axons by Wnt-frizzled signaling. Science 302:1984-1988.

Maison S, Liberman LD, Liberman MC (2016) Type II cochlear ganglion neurons do not drive the olivocochlear reflex: re-examination of the cochlear phenotype in peripherin knock-out mice. eNeuro 3:ENEURO. 0207-16.2016.

Mitchell B, Stubbs JL, Huisman F, Taborek P, Yu C, Kintner C (2009) The PCP pathway instructs the planar orientation of ciliated cells in the Xenopus larval skin. Curr Biol 19:924-929.

Montcouquiol M, Rachel RA, Lanford PJ, Copeland NG, Jenkins NA, Kelley MW (2003) Identification of Vangl2 and Scrb1 as planar polarity genes in mammals. Nature 423:173-177.

Morello F, Prasad AA, Rehberg K, Vieira de Sá R, Antón-Bolaños N, LeyvaDiaz E, Adolfs Y, Tissir F, López-Bendito G, Pasterkamp RJ (2015) Frizzled3 controls axonal polarity and intermediate target entry during striatal pathway development. J Neurosci 35:14205-14219.

Mulvaney JF, Yatteau A, Sun WW, Jacques B, Takubo K, Suda T, Yamada W, Dabdoub A (2013) Secreted factor R-Spondin 2 is involved in refinement of patterning of the mammalian cochlea. Dev Dyn 242:179-188.

Ohyama T, Groves AK (2004) Generation of Pax2-cre mice by modification of a Pax2 bacterial artificial chromosome. Genesis 38:195-199.

Onishi K, Zou Y (2017) Sonic Hedgehog switches on Wnt/planar cell polarity signaling in commissural axon growth cones by reducing levels of Shisa2. Elife 6:e25269.

Onishi K, Shafer B, Lo C, Tissir F, Goffinet AM, Zou Y (2013) Antagonistic functions of dishevelleds regulate Frizzled 3 endocytosis via filopodia tips in Wnt-mediated growth cone guidance. J Neurosci 33:19071-19085.

Qian D, Jones C, Rzadzinska A, Mark S, Zhang X, Steel KP, Dai X, Chen P (2007) Wnt5a functions in planar cell polarity regulation in mice. Dev Biol 306:121-133.

Qu Y, Glasco DM, Zhou L, Sawant A, Ravni A, Fritzsch B, Damrau C, Murdoch JN, Evans S, Pfaff SL, Formstone C, Goffinet AM, Chandrasekhar A, Tissir F
(2010) Atypical cadherins Celsr 1-3 differentially regulate migration of facial branchiomotor neurons in mice. J Neurosci 30:9392-9401.

Qu Y, Huang Y, Feng J, Alvarez-Bolado G, Grove EA, Yang Y, Tissir F, Zhou L, Goffinet AM (2014) Genetic evidence that Celsr3 and Celsr2, together with Fzd3, regulate forebrain wiring in a Vangl-independent manner. Proc Natl Acad Sci U S A 111:E2996-E3004.

Ryu YK, Collins SE, Ho HY, Zhao H, Kuruvilla R (2013) An autocrine Wnt5a-ror signaling loop mediates sympathetic target innervation. Dev Biol 377:79-89.

Schwenk F, Baron U, Rajewsky K (1995) A cre-transgenic mouse strain for the ubiquitous deletion of loxP-flanked gene segments including deletion in germ cells. Nucleic Acids Res 23:5080-5081.

Shafer B, Onishi K, Lo C, Colakoglu G, Zou Y (2011) Vangl2 promotes Wnt/planar cell polarity-like signaling by antagonizing Dvl1-mediated feedback inhibition in growth cone guidance. Dev Cell 20:177-191.

Shi F, Cheng YF, Wang XL, Edge AS (2010) Beta-catenin up-regulates Atoh1 expression in neural progenitor cells by interaction with an Atoh1 3' enhancer. J Biol Chem 285:392-400.

Sienknecht UJ, Anderson BK, Parodi RM, Fantetti KN, Fekete DM (2011) Non-cell-autonomous planar cell polarity propagation in the auditory sensory epithelium of vertebrates. Dev Biol 352:27-39.

Sittaramane V, Pan X, Glasco DM, Huang P, Gurung S, Bock A, Li S, Wang H, Kawakami K, Matise MP, Chandrasekhar A (2013) The PCP protein Vangl2 regulates migration of hindbrain motor neurons by acting in floor plate cells, and independently of cilia function. Dev Biol 382:400-412.

Stoller ML, Roman O Jr, Deans MR (2018) Domineering non-autonomy in Vangl1;Vangl2 double mutants demonstrates intercellular PCP signaling in the vertebrate inner ear. Dev Biol 437:17-26.

Stoller M, Roman O, Francl A, Deans M (2016) Analysis of Vangl1;Vangl2 double knockouts reveal conserved and unique polarity mechanisms in the developing utricle. Association for Research in Otolaryngology 39th Annual Midwinter Meeting, San Diego.

Struhl G, Casal J, Lawrence PA (2012) Dissecting the molecular bridges that mediate the function of frizzled in planar cell polarity. Development 139:3665-3674.

Tissir F, Goffinet AM (2013) Shaping the nervous system: role of the core planar cell polarity genes. Nat Rev Neurosci 14:525-535.

Torban E, Patenaude AM, Leclerc S, Rakowiecki S, Gauthier S, Andelfinger G, Epstein DJ, Gros P (2008) Genetic interaction between members of the Vangl family causes neural tube defects in mice. Proc Natl Acad Sci U S A 105:3449-3454

Wang Y, Nathans J (2007) Tissue/planar cell polarity in vertebrates: new insights and new questions. Development 134:647-658.

Wang Y, Thekdi N, Smallwood PM, Macke JP, Nathans J (2002) Frizzled-3 is required for the development of major fiber tracts in the rostral CNS. J Neurosci 22:8563-8573.

Wang Y, Guo N, Nathans J (2006) The role of Frizzled3 and Frizzled6 in neural tube closure and in the planar polarity of inner-ear sensory hair cells. J Neurosci 26:2147-2156.

Wu J, Mlodzik M (2008) The frizzled extracellular domain is a ligand for van Gogh/Stbm during nonautonomous planar cell polarity signaling. Dev Cell 15:462-469.

Yamamoto S, Nishimura O, Misaki K, Nishita M, Minami Y, Yonemura S, Tarui H, Sasaki H (2008) Cthrcl selectively activates the planar cell polarity pathway of Wnt signaling by stabilizing the Wnt-receptor complex. Dev Cell 15:23-36.

Yang Y (2012) Wnt signaling in development and disease. Cell Biosci 2:14.

Yang Y, Mlodzik M (2015) Wnt-Frizzled/planar cell polarity signaling: cellular orientation by facing the wind (Wnt). Annu Rev Cell Dev Biol 31:623-646.

Yin H, Copley CO, Goodrich LV, Deans MR (2012) Comparison of phenotypes between different Vangl2 mutants demonstrates dominant effects of the looptail mutation during hair cell development. PLoS One 7:e31988.

Zhang KD, Coate TM (2017) Recent advances in the development and function of Type II spiral ganglion neurons in the mammalian inner ear. Semin Cell Dev Biol 65:80-87.

Zou Y (2004) Wnt signaling in axon guidance. Trends Neurosci 27:528-532.

Zou Y (2012) Does planar cell polarity signaling steer growth cones? Curr Top Dev Biol 101:141-160. 